\section{A game changer in restorative dentistry}

G-ænial Universal Injectable (GUI) has changed the composite market since its launch thanks to its practicality, optimal physical properties which are stronger than many paste

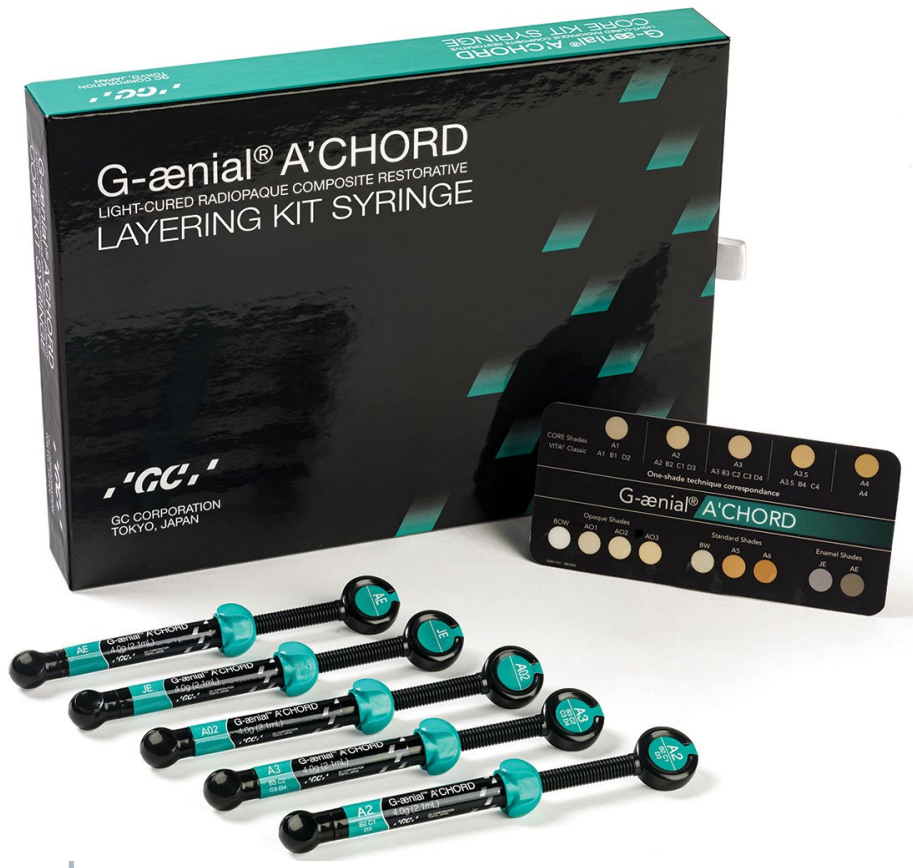

composites, and long-term aesthetics. Its injectable, highly thixotropic viscosity allows excellent adaptation to the cavity walls while keeping its shape during placement, making it possible to directly create the final anatomy while injecting in the cavity. Its strength and wear resistance makes it perfectly suited for use in posterior restorations without capping and in the Injection Moulding Technique (IMT).

GC's unique Full-coverage Silane Coating (FSC) technology improves the coupling between the fillers and the matrix making it injectable and shapeable at the same time. It adapts perfectly to the cavity floor without slumping or sticking to instruments.

Thanks to G-ænial Universal Injectable's flexural strength and wear resistance, it is ideal for direct restoration of worn tooth surfaces or the creation of direct veneers using the IMT. Its high thixotropy enables it to keep its shape or to spread on the tooth surface depending on the constraint applied on the material. It is indicated for all restoration classes and to build margins or restore occlusal surfaces, cusp by cusp.

As well as optimal handling, this composite offers great aesthetic possibilities with a wide range of shades in three levels of translucency, including opaque and enamel shades as well as bleach shades. The ultra-fine barium particles with a very homogeneous dispersion help to achieve great gloss retention for a long-lasting shine. Its high radiopacity of $252 \% \mathrm{Al}$ makes it ideal for follow-up of restorations and easy detection of secondary caries.

G-ænial Universal Injectable offers easier restorative options without compromising on durability or aesthetics. Now, with the availability of single-use unitips, clinicians can choose the best delivery for their patients and practice.

For more information on G-ænial Universal Injectable contact GC UK Ltd on 01908 218999, email info.uk@gc.dental or visit www.gceurope.com/products/gaenialuniversalinjectable.

\title{
Long-lasting results you and your patients will value
}
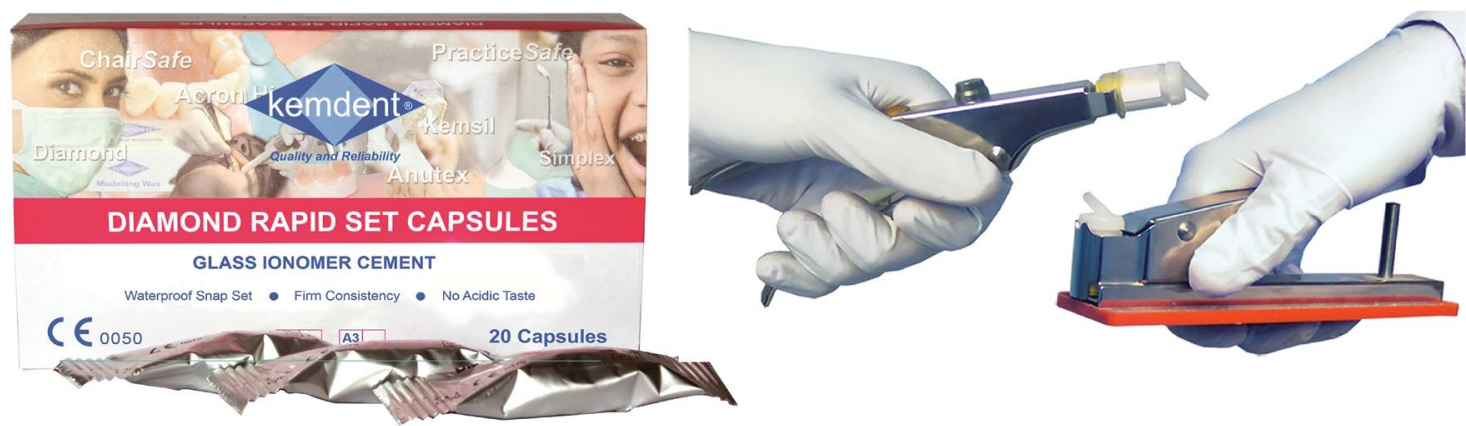

Kemdent Diamond Rapid Set Glass Ionomer Cement Capsules provide extreme durability for long-lasting results you and your patients will love.

They are ideal for non-load-bearing Class I and Class II restorations, as well as for deciduous teeth, the sandwich technique and core build-ups. The innovative formula renders the material resistant to saliva almost immediately, minimising the risk of contamination from moisture for maximum material strength after six months, a compressive strength of $350 \mathrm{MPa}$ is achieved.
In addition, the capsules are individually wrapped in easyopen foils, facilitating single-dose dentistry and optimising both efficiency and hygiene in the surgery. They even release fluoride to provide a level of protection against caries.

Looking for durable restorative results you and your patients can trust? Discover Kemdent Diamond Rapid Set Glass Ionomer Cement Capsules.

For more information, visit www.kemdent.co.uk. 